\title{
Study of $\Gamma$-hyperrings by fuzzy hyperideals with respect to a t-norm
}

\author{
Krisanthi Naka \\ Department of Mathematics and \\ Computer Sciences, \\ Faculty of Natural Sciences, \\ University of Gjirokastra, Albania \\ email: anthinaka@yahoo.com \\ Serkan Onar \\ Yildiz Technical University, \\ Department of Mathematics, \\ Davutpaşa-Istanbu, Turkey \\ email: serkan10ar@gmail.com
}

\author{
Kostaq Hila \\ Department of Mathematical \\ Engineering, Polytechnic \\ University of Tirana, Albania \\ email: kostaq_hila@yahoo.com
Bayram Ali Ersoy
Yildiz Technical University,
Davutpaşa-Istanbu, Turkey
email: ersoya@gmail.com \\ Department of Mathematics,
}

\begin{abstract}
In this paper, we study the $\Gamma$-hyperrings via T-fuzzy hyperideals. By means of the use of a triangular norm $\mathrm{T}$, we define, characterize and study the T-fuzzy left and right hyperideals, T-fuzzy quasihyperideal and bi-hyperideal in $\Gamma$-hyperrings and some related properties are investigated. Regular $\Gamma$-hyperrings are characterized in terms of $\mathrm{T}$ fuzzy quasi-hyperideal and T-fuzzy bi-hyperideal. We also introduce the $\mathrm{T}$ - $(\lambda, \mu)$-fuzzy bi-hyperideals in $\Gamma$-hyperrings and investigate some of their properties.
\end{abstract}

\section{Introduction and preliminaries}

The applications of mathematics in other disciplines, for example in informatics, play a key role and they represent, in the last decades, one of the purposes

2010 Mathematics Subject Classification: 16Y99 16D25 20N20 08A72

Key words and phrases: $\Gamma$-hyperrings, t-norm, T-fuzzy (resp. left, right) hyperideal, Tfuzzy quasi(bi)-hyperideal 
of the study of the experts of Hyperstructures Theory all over the world. Hyperstructures, as a natural extension of classical algebraic structures, in particular hypergroups, were introduced in 1934 by a French mathematician, Marty, at the 8th Congress of Scandinavian Mathematicians [29]. Since then, a lot of papers and several books have been written on this topic. Nowadays, hyperstructures have a lot of applications to several domains of mathematics and computer science(see $[10,24,25,33,41,44])$ and they are studied in many countries of the world. This theory has been subsequently developed by Corsini [11, 9, 10], Mittas [27, 28], Stratigopoulos [40] and by various authors. Basic definitions and propositions about the hyperstructures are found in $[9,10,15,29,41]$. Krasner [22] has studied the notion of hyperfields, hyperrings, and then some researchers, namely, Ameri [2], Dasic [12], Davvaz [14, 30, 13], Gontineac [19], Massouros [26], Pianskool et al. [34], Sen and Dasgupta [38], Vougiouklis [41, 42] and others followed him.

Hyperrings are essentially rings with approximately modified axioms. There are different notions of hyperrings $(R,+, \cdot)$. If the addition + is a hyperoperation and the multiplication - is a binary operation, then the hyperring is called Krasner (additive) hyperring [22]. Rota [35] introduced a multiplicative hyperring, where + is a binary operation and the multiplication - is a hyperoperation. De Salvo [37] studied hyperrings in which the additions and the multiplications were hyperoperations. These hyperrings were also studied by Barghi [7] and by Asokkumar and Velrajan [5, 6]. In 2007, Davvaz and Leoreanu-Fotea [15] published a book titled Hyperring Theory and Applications.

$\Gamma$-rings were introduced by Nobusawa [31] as an algebraic tool for observing the relationship between the groups of homomorphisms hom $(\mathrm{B}, \mathrm{C})$ and hom $(C, B)$ of commutative groups B and $C$. The class of $\Gamma$-rings contains not only all rings but also Hestenes ternary rings. Barnes [8] weakened slightly the conditions in the definition of $\Gamma$-ring in the sense of Nobusawa. The study of $\Gamma$-hyperrings as a generalization of rings, ternary rings, $\Gamma$-rings was initiated by Ameri et. al. [4].

After the introduction of the concept of fuzzy sets by Zadeh in 1965 [45], it has found manifold applications in the field of mathematics and related areas. This provides sufficient motivations for researchers to review various concepts and results from the realm of abstract algebra to a broader framework of fuzzy setting. The study of fuzzy algebraic structures was started with the introduction of the concepts of fuzzy subgroups by Rosenfeld [36]. The study of fuzzy hyperstructures is an interesting research topic of fuzzy sets. There is a considerable amount of work on the connections between fuzzy sets and 
hyperstructures. Davvaz [17] introduced the concept of fuzzy hyperideals in a semihypergroup. In 2009, Davvaz [18] gave the concept of fuzzy hyperideals in ternary semihyperrings. The relationships between the fuzzy sets and algebraic hyperstructures have been considered by Corsini, Davvaz, Leoreanu, Zhan, Zahedi, Ameri, Cristea and many other researchers. For more on fuzzy hyperstructures one can see [16]. The fuzzy hyperring notion is defined and studied in [23]. The fuzzy $\Gamma$-hyperring notion is defined and studied in [4, 43].

On the other hand, in 1960, Schweizer and Sklar [39] introduced the notion of triangular norm ( $\mathrm{t}$-norm) and triangular conorm ( $\mathrm{t}$-conorm) in order to generalize the ordinary triangle inequality in a metric space to the more general probabilistic metric space. Using t-norm, Anthony and Sherwood [1] first redefined Rosenfeld's [36] notion of fuzzy groups. Since then $t$-norm has played an important role in fuzzy algebra. In application, $t$-norm $T$ and $t$-conorm $S$ are the functions that map the unit square into the unit interval. In fuzzy sets theory, triangular norm ( $\mathrm{t}$-norm) is extensively used to model the logical connective: conjunction (AND). There are many applications of triangular norms in several fields of mathematics and artificial intelligence [21].

In this paper, we inquire further into the properties on some kind fuzzy hyperideals and we study the $\Gamma$-hyperrings via $T$-fuzzy hyperideals. By means of the use of a triangular norm $\mathrm{T}$, we define, characterize and study the T-fuzzy left and right hyperideals, T-fuzzy quasi-hyperideal and bi-hyperideal in $\Gamma$-hyperrings and some related properties are investigated. We compare fuzzy hyperideal to T-fuzzy hyperideals. We have shown that $\Gamma$-hyperring is regular if and only if intersection of any T-fuzzy right hyperideal with T-fuzzy left hyperideal is equal to its product. We introduce the notion of T-fuzzy quasi-hyperideal and T-fuzzy bi-hyperideal. We discuss some of its properties. We have shown that the meet of T-fuzzy right and T-fuzzy left ideal is a Tfuzzy quasi hyperideal of a $\Gamma$-hyperring. We characterize regular $\Gamma$-hyperring with T-fuzzy quasi-hyperideal and T-fuzzy bi-hyperideal. We also introduce the $T$ - $(\lambda, \mu)$-fuzzy bi-hyperideals in $\Gamma$-hyperrings and investigate some of their properties.

Recall first the basic terms and definitions from the hyperstructure theory. In a classical algebraic structure, the composition of two elements is an element, while in an algebraic hyperstructure, the composition of two elements is a set.

A map $\circ: \mathrm{H} \times \mathrm{H} \rightarrow \mathcal{P}^{*}(\mathrm{H})$ is called hyperoperation or join operation on the set $\mathrm{H}$, where $\mathrm{H}$ is a non-empty set and $\mathcal{P}^{*}(\mathrm{H})=\mathcal{P}(\mathrm{H}) \backslash\{\emptyset\}$ denotes the set of all nonempty subsets of $\mathrm{H}$. A hyperstructure is called the pair $(\mathrm{H}, \mathrm{o})$ where $\circ$ is a hyperoperation on the set $\mathrm{H}$. A hyperstructure $(\mathrm{H}, \mathrm{o})$ is called a 
semihypergroup if for all $x, y, z \in H,(x \circ y) \circ z=x \circ(y \circ z)$, which means that

$$
\bigcup_{u \in x \circ y} u \circ z=\bigcup_{v \in y \circ z} x \circ v \text {. }
$$

If $x \in H$ and $A, B$ are nonempty subsets of $H$, then

$$
A \circ B=\bigcup_{a \in A, b \in B} a \circ b, A \circ x=A \circ\{x\} \text {, and } x \circ B=\{x\} \circ B \text {. }
$$

A non-empty subset $\mathrm{B}$ of a semihypergroup $\mathrm{H}$ is called a sub-semihypergroup of $\mathrm{H}$ if $\mathrm{B} \circ \mathrm{B} \subseteq \mathrm{B}$ and $\mathrm{H}$ is called in this case super-semihypergroup of $\mathrm{B}$. Let $(\mathrm{H}, \circ)$ be a semihypergroup. Then $\mathrm{H}$ is called a hypergroup if it satisfies the reproduction axiom, for all $\mathrm{a} \in \mathrm{H}, \mathrm{a} \circ \mathrm{H}=\mathrm{H} \circ \mathrm{a}=\mathrm{H}$. An element $e$ in a semihypergroup $\mathrm{H}$ is called identity if

$$
x \circ e=e \circ x=\{x\}, \forall x \in H .
$$

An element 0 in a semihypergroup $\mathrm{H}$ is called zero element if

$$
x \circ 0=0 \circ x=\{0\}, \forall x \in H .
$$

A non-empty set $\mathrm{H}$ with a hyperoperation + is said to be a canonical hypergroup if the following conditions hold:

1. for every $x, y \in H, x+y=y+x$,

2. for every $x, y, z \in H, x+(y+z)=(x+y)+z$,

3. there exists $0 \in \mathrm{H}$, (called neutral element of $\mathrm{H}$ ) such that $0+x=\{x\}=$ $x+0$ for all $x \in H$,

4. for every $x \in H$, there exists a unique element denoted by $-x \in H$ such that $0 \in x+(-x) \cap(-x)+x$,

5. for every $x, y, z \in H, z \in x+y$ implies $y \in-x+z$ and $x \in z-y$.

A comprehensive review of the theory of hypergroups appears in [9]. For any subset $A$ of a canonical hypergroup $H,-A$ denotes the set $\{-a: a \in A\}$. A non-empty subset $\mathrm{N}$ of a canonical hypergroup of $\mathrm{H}$ is called a subcanonical hypergroup of $\mathrm{H}$ if $\mathrm{N}$ is a canonical hypergroup under the same hyperoperation as that of $H$. Equivalently, for every $x, y \in N, x-y \subseteq N$. In particular, for any $x \in N, x-x \subseteq N$. Since $0 \in x-x$, it follows that $0 \in N$.

There are several kinds of hyperrings that can be defined on a non-empty set R. In what follows, we shall consider one of the most general types of hyperrings. The definition of a hyperring given below is equivalent to one formulated by De Salvo [37] (see Corsini [9]). 
Definition 1 A hyperring is a triple $(\mathrm{R},+, \cdot)$, where $\mathrm{R}$ is a non-empty set with a hyperaddition + and a hypermultiplication - satisfying the following axioms:

1. $(\mathrm{R},+)$ is a canonical hypergroup,

2. ( $\mathrm{R}, \cdot)$ is a semihypergroup such that $\mathrm{x} \cdot 0=0 \cdot \mathrm{x}=0$ for all $\mathrm{x} \in \mathrm{R}$, (i.e, 0 is a bilaterally absorbing element),

3. The hypermultiplication - is distributive with respect to the hyperoperation + . That is, for every $x, y, z \in R, x \cdot(y+z)=x \cdot y+x \cdot z$, and $(x+y) \cdot z=x \cdot z+y \cdot z$.

Definition 2 [37, 9] A non-empty subset $\mathrm{R}^{\prime}$ of $\mathrm{R}$ is called a subhyperring of $(\mathrm{R},+, \cdot)$ if $\left(\mathrm{R}^{\prime},+\right)$ is a subhypergroup of $(\mathrm{R},+)$ and $\forall x, y \in \mathrm{R}^{\prime}, x \cdot \mathrm{y} \in \mathcal{P}^{*}\left(\mathrm{R}^{\prime}\right)$.

Example 1 Let $\mathrm{R}=\{0,1\}$ be a set with two hyperoperations defined as follows:

\begin{tabular}{|c|c|c|c|c|}
\hline & 0 & 1 & . & 1 \\
\hline 0 & $\{0\}$ & $\{0,1\}$ & 0 & $\{0\}$ \\
\hline 1 & $\{0,1\}$ & $\{0,1\}$ & 1 & $\{0,1\}$ \\
\hline
\end{tabular}

Clearly, $(\mathrm{R},+, \cdot)$ is a hyperring.

Example 2 Let $\mathrm{R}=\{0, \mathrm{a}, \mathrm{b}\}$ be a set with two hyperoperations defined as follows.

\begin{tabular}{c|ccccc|ccc}
+ & 0 & $a$ & $b$ & & 0 & $a$ & $b$ \\
\hline 0 & $\{0\}$ & $\{a\}$ & $\{b\}$ & & 0 & $\{0\}$ & $\{0\}$ & $\{0\}$ \\
$a$ & $\{a\}$ & $\{a, b\}$ & $R$ & & $a$ & $\{0\}$ & $R$ & $R$ \\
$b$ & $\{b\}$ & $R$ & $\{a, b\}$ & & $b$ & $\{0\}$ & $R$ & $R$
\end{tabular}

Clearly, $(\mathrm{R},+, \cdot)$ is a hyperring.

Example 3 Let $(\mathrm{R},+, \cdot)$ be a hyperring. Then $\left(\mathrm{M}_{\mathrm{n}}(\mathrm{R}), \oplus, \odot\right)$ is a hyperring, where $\mathrm{M}_{\mathrm{n}}(\mathrm{R})$ is the set of all $\mathrm{n} \times \mathrm{n}$ matrices over $\mathrm{R}$ for some natural number $\mathrm{n}$ and the hyperoperations $\oplus$ and $\odot$ are defined as follows:

For $x=\left(x_{i j}\right), y=\left(y_{i j}\right) \in M_{n}(R), x \oplus y=\left\{z \in M_{n}(R): z=\left(z_{i j}\right), z_{i j} \in\right.$ $\left.x_{i j}+y_{i j}, 1 \leq i, j \leq n\right\}$ and $x \odot y=\left\{z \in M_{n}(R): z=\left(z_{i j}\right), z_{i j} \in \sum_{k=1}^{n} x_{i k} \cdot y_{k j}, 1 \leq\right.$ $i, j \leq n\}$.

Definition 3 [37, 9] Let $(\mathrm{R},+, \cdot)$ be a hyperring. A non-empty subset $\mathrm{A}$ of $\mathrm{R}$ is called a hyperideal of $\mathrm{R}$ if $(\mathrm{A},+)$ is a subhypergroup of $(\mathrm{R},+)$ and $\forall \mathrm{x} \in$ $\mathrm{R}, \forall \mathrm{y} \in A$, both $\mathrm{x} \cdot \mathrm{y}$ and $\mathrm{y} \cdot \mathrm{x}$ are elements of $\mathcal{P}^{*}(A)$. 
Let $R$ and $\Gamma$ be two non-empty sets. A map from $R \times \Gamma \times R \rightarrow P^{*}(R)$ will be called a $\Gamma$-hypermultiplication in $R$ and is denoted by $(\cdot)_{\Gamma}$. The result of this $\Gamma$-hypermultiplication for every two elements $a, b \in R$ and every element $\gamma \in \Gamma$ is denoted by $a \gamma b$. In the following we give the definition of $\Gamma$-hyperrings in a different way.

Definition 4 (cf. [4]) A $\Gamma$-hyperring is called a five tuple $\left(\mathrm{R}, \Gamma,+, \oplus,(\cdot)_{\Gamma}\right)$ where $\mathrm{R}, \Gamma$ are nonempty sets, + is the hyperaddition in $\mathrm{R}, \oplus$ is the hyperaddition in $\Gamma,(\cdot)_{\Gamma}$ is a $\Gamma$-hypermultiplication in $\mathrm{R}$, such that:

1. $(\mathrm{R},+)$ is a canonical hypergroup;

2. $(\Gamma, \oplus)$ is a canonical hypergroup;

3. $\forall(x, y, z, \alpha) \in R^{3} \times \Gamma,(x+y) \alpha z=x \alpha z+y \alpha z, x \alpha(y+z)=x \alpha y+x \alpha z$;

4. $\forall(x, y, \alpha, \beta) \in R^{2} \times \Gamma^{2}, x(\alpha \oplus \beta) y=x \alpha y+x \beta y$;

5. $\forall(x, y, z, \alpha, \beta) \in R^{3} \times \Gamma^{2},(x \alpha y) \beta z=x \alpha(y \beta z)$.

Definition $5[20]$ A $\Gamma$-semihypergroup $\mathrm{R}$ is called an ordered pair $\left(\mathrm{R},(\cdot)_{\Gamma}\right)$ where $\mathrm{R}$ and $\Gamma$ are nonempty sets and $(\cdot)_{\Gamma}$ is a $\Gamma$-hypermultiplication on $\mathrm{R}$ which satisfies the following property: $\forall(a, b, c, \alpha, \beta) \in R^{3} \times \Gamma^{2},(a \alpha b) \beta c=a \alpha(b \beta c)$.

Definition 6 An weakly $\Gamma$-hyperring is called any triple $\left(\mathrm{R},+,(\cdot)_{\Gamma}\right)$ where $\mathrm{R}, \Gamma$ are nonempty sets, + is the hyperaddition in $\mathrm{R},(\cdot)_{\Gamma}$ is a $\Gamma$-hypermultiplication in $\mathrm{R}$, such that:

1. $(\mathrm{R},+)$ is a canonical hypergroup;

2. $\left(\mathrm{R},(\cdot)_{\Gamma}\right)$ is a $\Gamma$-semihypergroup;

3. $\forall(x, y, z, \alpha) \in R^{3} \times \Gamma,(x+y) \alpha z=x \alpha z+y \alpha z, x \alpha(y+z)=x \alpha y+x \alpha z$.

Examples of $\Gamma$-hyperrings can be found in $[4,43]$. It is clear tht every hyperring is a $\Gamma$-hyperring.

In what follows, unless otherwise stated, an weakly $\Gamma$-hyperring $(R,+, \Gamma)$ always denotes a $\Gamma$-hyperring.

Definition 7 [4] A non-empty subset $\mathrm{R}^{\prime}$ of $(\mathrm{R},+, \Gamma)$ is called a sub- $\Gamma$-hyperring of $\mathrm{R}$ if $\left(\mathrm{R}^{\prime},+\right)$ is a subhypergroup of $(\mathrm{R},+)$ and $\forall x, \mathrm{y} \in \mathrm{R}^{\prime}, \gamma \in \Gamma, \mathrm{x} \gamma \mathrm{y} \subseteq \mathcal{P}^{*}\left(\mathrm{R}^{\prime}\right)$. 
Definition 8 [4] Let $(\mathrm{R},+, \Gamma)$ be a $\Gamma$-hyperring. A non-empty subset $\mathrm{A}$ of $\mathrm{R}$ is called a right (left) hyperideal of $\mathrm{R}$ if $(A,+)$ is a subhypergroup of $(R,+)$ and $\mathrm{A} \Gamma \mathrm{R} \subseteq \mathrm{A}(\mathrm{R} \Gamma \mathrm{A} \subseteq \mathrm{A}) . A$ is called a hyperideal if it is both a left and a right hyperideal of $\mathrm{R}$.

Definition 9 A non-empty subset $\mathrm{Q}$ of $(\mathrm{R},+, \Gamma)$ is said to be a quasi-hyperideal of $\mathrm{R}$ if $(\mathrm{Q},+)$ is a subhypergroup of $(\mathrm{R},+)$ and $\mathrm{Q} \Gamma \mathrm{R} \cap \mathrm{R} \Gamma \mathrm{Q} \subseteq \mathrm{Q}$. A non-empty subset $\mathrm{B}$ of $\mathrm{R}$ is said to be a bi-hyperideal of $(\mathrm{R},+, \Gamma)$ if $(\mathrm{B},+)$ is a subhypergroup of $(\mathrm{R},+)$ and $\mathrm{B} \Gamma \mathrm{R} \Gamma \mathrm{B} \subseteq \mathrm{B}$.

Definition 10 [32] An element $\mathrm{a} \in \mathrm{R}$ is said to be regular if $\mathrm{a} \in \mathrm{a} \Gamma \mathrm{R} \Gamma \mathrm{a}$. That is, there exist an element $\mathrm{b} \in \mathrm{R}$ and $\alpha, \beta \in \Gamma$ such that $\mathrm{a} \in \mathrm{a} \alpha \mathrm{b} \beta \mathrm{a}$. A $\Gamma$-hyperring $\mathrm{R}$ is said to be regular if every element of $\mathrm{R}$ is regular.

A mapping $\mu: X \rightarrow[0,1]$, where $X$ is an arbitrary non-empty set and is called a fuzzy set in $X$. For $\alpha \in[0,1]$, the set $U(\mu ; \alpha)=\{x \in X \mid \mu(x) \geq \alpha\}$ is called level set of $\mu$. A fuzzy set $\mu$ in a $\Gamma$-hyperring $\mathrm{R}$ is called a fuzzy left (resp. right) hyperideal of $\mathrm{R}$ if it satisfies:

- $\inf _{a \in x-y} \mu(a) \geq \min \{\mu(x), \mu(y)\}$,

- $\inf _{a \in x \gamma y} \mu(a) \geq \mu(y)\left(\right.$ resp. $\left.\inf _{a \in x \gamma y} \mu(a) \geq \mu(x)\right)$ for all $x, y \in R$ and $\gamma \in \Gamma$.

A fuzzy set $\mu$ in a $\Gamma$-hyperring $R$ is called a fuzzy hyperideal of $\mathrm{R}$ if $\mu$ is both a fuzzy left and a fuzzy right hyperideal of $\mathrm{R}[4]$.

A triangular norm (briefly, t-norm) (cf. Schweizer and Sklar [39]) is a function $T:[0,1] \times[0,1] \rightarrow[0,1]$ satisfying for every $x, y, z \in[0,1]$ the following conditions:

(T1) $\mathrm{T}(x, y)=\mathrm{T}(y, x)$ (commutative),

(T2) $\mathrm{T}(x, y) \leq \mathrm{T}(x, z)$ if $y \leq z$ (monotone in the right factor),

(T3) $\mathrm{T}(x, \mathrm{~T}(\mathrm{y}, z))=\mathrm{T}(\mathrm{T}(x, y), z)$ (associative),

(T4) $\mathrm{T}(x, 1)=x$ (having 1 as a right identity).

These four axioms are independent in the sense that none of them can be deduced from the other three. Obviously, the function min defined on $[0,1] \times$ $[0,1]$ is a $t$-norm. Other $t$-norms which are frequently encountered in the study of probabilistic spaces are $T_{m}$ and $T_{p}$ defined by $T_{m}(a, b)=\max (a+b-$ $1,0), T_{p}(a, b)=a b$ for every $a, b \in[0,1]$. Replacing 1 by 0 in condition (T4) we obtain the concept of triangular conorm ( $t$-conorm). In general every $t$ norm $\mathrm{T}$ satisfies the following conditions: 
(i) $\mathrm{T}(x, 0)=0 ; \mathrm{T}(0,0)=0$ and $\mathrm{T}(1,1)=1$;

(ii) $\mathrm{T}(x, y) \leq \min (x, y), \forall x, y \in[0,1]$.

For a t-norm $\mathrm{T}$ on $[0,1]$, we denote it $\mathrm{E}_{\mathrm{T}}=\{\alpha \in[0,1] \mid \mathrm{T}(\alpha, \alpha)=\alpha\}$.

Let $T_{1}$ and $T_{2}$ be two t-norms. $T_{2}$ is said to be dominate $T_{1}$ and write $T_{1} \ll T_{2}$ if for all $a, b, c, d \in[0,1], T_{1}\left(T_{2}(a, c), T_{2}(b, d)\right) \leq T_{2}\left(T_{1}(a, b), T_{1}(c, d)\right)$ and $T_{1}$ is said weaker then $T_{2}$ or $T_{2}$ is stronger then $T_{1}$ and write $T_{1} \leq T_{2}$ if for all $x, y \in[0,1], T_{1}(x, y) \leq T_{2}(x, y)$. Since a triangular norm $T$ is a generalization of the minimum function, Anthony and Sherwood in [1] replaced the axiom $\min \{\mu(x), \mu(y)\} \leq \mu(x y)$ occurring in the definition of a fuzzy subgroup by the inequality $T(\mu(x), \mu(y)) \leq \mu(x y)$.

Definition 11 Let $\mu, \lambda$ be the fuzzy subsets of a set $\mathrm{X}$. A fuzzy subset $\mu \cap \lambda$ is defined as $(\mu \cap \lambda)(x)=\min (\mu(x), \lambda(x))$.

Definition 12 Let $\mu, \lambda$ be the fuzzy subsets of a set $\mathrm{X}$. A fuzzy subset $\mu \wedge \lambda$ is defined as $(\mu \wedge \lambda)(x)=\mathrm{T}(\mu(\mathrm{x}), \lambda(\mathrm{x}))$.

Definition 13 Let $\mu, \lambda$ be the fuzzy subsets of a set $\mathrm{X}$. The product of the fuzzy subset $\mu$ and $\lambda$ is defined as $(\mu \circ \lambda)(x)=\sup _{x \in y \gamma z} T(\mu(y), \lambda(z)), \gamma \in \Gamma$.

\section{T-fuzzy right and left hyperideals in $\Gamma$-hyperrings}

In this section, we introduce the notions of T-fuzzy left hyperideal and T-fuzzy right hyperideal in $\Gamma$-hyperrings and some properties of them are studied. Also, the regular $\Gamma$-hyperrings are studied in terms of T-fuzzy left hyperideals and T-fuzzy right hyperideals.

Definition 14 A fuzzy set $\mu$ in a $\Gamma$-hyperring $\mathrm{R}$ is called a fuzzy left (resp. right) hyperideal of $\mathrm{R}$ with respect to a $\mathrm{t}$-norm $\mathrm{T}$ (briefly, a $\mathrm{T}$-fuzzy left (resp. right) hyperideal of $\mathrm{R}$ ) if it satisfies:

1. $\inf _{a \in x-y} \mu(a) \geq T(\mu(x), \mu(y))$,

2. $\inf _{a \in x \gamma y} \mu(a) \geq \mu(y)\left(\right.$ resp. $\left.\inf _{a \in x \gamma y} \mu(a) \geq \mu(x)\right)$

for all $x, y \in \mathrm{R}$ and $\gamma \in \Gamma$.

Remark 1 1. If we take $\mathrm{t}$-norm as min-norm, T-fuzzy right hyperideal coincides with fuzzy right hyperideal [3].

2. T-fuzzy hyperideal is both T-fuzzy right and left hyperideal. 
Lemma 1 Let $(\mathrm{R},+, \Gamma)$ be a $\Gamma$-hyperring. Every fuzzy right hyperideal of $\mathrm{R}$ is a $\mathrm{T}$-fuzzy right hyperideal.

Proof. Let $\mu$ be a fuzzy right hyperideal of R. Then $\inf _{a \in x-y} \mu(a) \geq \min \{\mu(x)$, $\mu(y)\} \geq T(\mu(x), \mu(y)\}$ and $\inf _{a \in x \gamma y} \mu(a) \geq \mu(x)$ for all $x, y \in R$ and $\gamma \in \Gamma$. Hence $\mu$ is a T-fuzzy hyperideal.

Corollary 1 Let $(\mathrm{R},+, \Gamma)$ be a hyperring. If $\mathrm{A}$ is a right hyperideal of $\mathrm{R}$, then $\chi_{\mathrm{A}}$ is a $\mathrm{T}$-fuzzy right hyperideal.

Proof. Let $A$ be a right hyperideal of a $\Gamma$-hyperring $R$. Then $\chi_{A}$ is a fuzzy right hyperideal. Therefore by Lemma $1, \chi_{A}$ is a $T$-fuzzy right hyperideal.

Note: Every T-fuzzy right (resp. left) hyperideal need not be a fuzzy hyperideal by the following examples.

Example 4 Let $\mathrm{R}=\{0, \mathrm{a}, \mathrm{b}, \mathrm{c}, \mathrm{d}\}$ be a set with two hyperoperations defined as follows.

\begin{tabular}{|c|c|c|c|c|c|c|c|c|c|c|c|}
\hline+ & 0 & $a$ & $\mathrm{~b}$ & $c$ & $\mathrm{~d}$ & & 0 & $a$ & $\mathrm{~b}$ & c & $\mathrm{d}$ \\
\hline 0 & 0 & $a$ & $\mathrm{~b}$ & $c$ & $\mathrm{~d}$ & 0 & 0 & 0 & 0 & 0 & 0 \\
\hline$a$ & $a$ & $\{0, a\}$ & $\mathrm{b}$ & $c$ & $d$ & $a$ & 0 & $\{0, a\}$ & $\{0, a\}$ & $\{0, a\}$ & $\{0, a\}$ \\
\hline$b$ & b & $\mathrm{b}$ & $\{0, a\}$ & $d$ & $c$ & $b$ & 0 & $\{0, a\}$ & $\{0, a\}$ & $\{0, a\}$ & $\{0, a\}$ \\
\hline c & $c$ & $c$ & $\mathrm{~d}$ & $\{0, a\}$ & $b$ & $c$ & 0 & $\{0, a\}$ & $\{0, a\}$ & $\{0, a\}$ & $\{0, a\}$ \\
\hline$d$ & d & $d$ & $c$ & $\mathrm{~b}$ & $\{0, a\}$ & $d$ & 0 & $\{0, a\}$ & $\{0, a\}$ & $\{0, a\}$ & $\{0, a\}$ \\
\hline
\end{tabular}

Clearly, $(\mathrm{R},+, \cdot)$ is a hyperring. Let $\Gamma=\{0, \mathrm{a}, \mathrm{b}\}$ be an hyperideal of $\mathrm{R}$. Then $\mathrm{R}$ is a $\Gamma$-hyperring. Define $\mu: \mathrm{R} \rightarrow[0,1]$ on $\mathrm{R}$ as follows:

$$
\mu(0)=0.8, \mu(a)=0.6, \mu(b)=0.5, \mu(c)=0.4, \mu(d)=0.3
$$

It can be easily verified that $\mu$ is a $\mathrm{T}$-fuzzy right hyperideal under $\mathrm{T}_{\mathrm{p}}$. But $\mu$ is not a fuzzy hyperideal of $\mathrm{R}$.

Example 5 [4] Let $\mathrm{I}_{1} \subset \mathrm{I}_{2} \subset \ldots . \subset \mathrm{I}_{\mathrm{n}} \subset \ldots$ be a strictly increasing sequence of left hyperideals of an arbitrary $\Gamma$-hyperring $\mathrm{R}$ and $\left\{\mathrm{t}_{\mathbf{j}}\right\}_{j=1}^{\infty}$ be a strictly increasing sequence in $[0,1]$. Define $\mu$ in $\mathrm{R}$ as follows:

$$
\begin{gathered}
\mu(\mathrm{x})=\mathrm{t}_{j} \text { if } \mathrm{x} \in \mathrm{I}_{\mathrm{j}} \backslash \mathrm{I}_{\mathrm{j}-1}, \text { where } \mathrm{t}_{j-1}<\mathrm{t}_{j}, \mathrm{j}=1,2, \ldots \text { and } \mu(\mathrm{x})=0 \text {, if } \\
x \in R \backslash \bigcup_{j=1}^{\infty} \mathrm{I}_{j}
\end{gathered}
$$


It can be easily verified that $\mu$ is a $\mathrm{T}$-fuzzy right hyperideal under $\mathrm{T}_{\mathrm{p}}$. But $\mu$ is not a fuzzy hyperideal of $\mathrm{R}$, it is only a left fuzzy hyperideal of $\mathrm{R}$.

Theorem 1 Let $(\mathrm{R},+, \Gamma)$ be a $\Gamma$-hyperring. If $\mu, \lambda$ are $\mathrm{T}$-fuzzy right hyperideals of $\mathrm{R}$, then $\mu \wedge \lambda$ is a $\mathrm{T}$-fuzzy right hyperideal of $\mathrm{R}$.

Proof. Let $x, y \in R, \gamma \in \Gamma$,

$$
\begin{aligned}
\inf _{a \in x-y}(\mu \wedge \lambda)(a) & \geq T\left(\inf _{a \in x-y} \mu(a), \inf _{a \in x-y} \lambda(a)\right) \\
& \geq T(T(\mu(x), \mu(y)), T(\lambda(x), \lambda(y))) \\
& =T(T(T(\mu(x), \mu(y)), \lambda(x)), \lambda(y)) \\
& \geq T(T(T(\mu(x), \lambda(x)), \mu(y), \lambda(y))) \\
& =T(T(\mu(x), \lambda(x)), T(\mu(y), \lambda(y))) \\
& =T((\mu \wedge \lambda)(x),(\mu \wedge \lambda)(y)) .
\end{aligned}
$$

Since $\inf _{a \in x \gamma y} \mu(a) \geq \mu(x)$ and $\inf _{a \in x \gamma y} \lambda(a) \geq \lambda(x)$, we have $T\left(\inf _{a \in x \gamma y} \mu(a), \inf _{a \in x \gamma y} \lambda(a)\right)$ $\geq T(\mu(x), \lambda(x))$. Then $\inf _{a \in x \gamma y}(\mu \wedge \lambda)(a) \geq(\mu \wedge \lambda)(x)$. Thus $\mu \wedge \lambda$ is a T-fuzzy right hyperideal of $R$.

Corollary 2 Let $(\mathrm{R},+, \Gamma)$ be a $\Gamma$-hyperring. If $\mu, \lambda$ are fuzzy right hyperideals of $\mathrm{R}$, then $\mu \cap \lambda$ is a fuzzy right hyperideal of $\mathrm{R}$.

Proof. By taking min as t-norm $\mathrm{T}$ in Theorem 1, we get the required result.

Lemma 2 Let $(\mathrm{R},+, \Gamma)$ be a hyperring. $\mathrm{R}$ is regular if and only if $\mathrm{A} \Gamma \mathrm{B}=\mathrm{A} \cap \mathrm{B}$ for any right hyperideal $\mathrm{A}$ and left hyperideal $\mathrm{B}$ of $\mathrm{R}$.

Proof. Let $(R,+, \Gamma)$ be a regular $\Gamma$-hyperring and $A, B$ be the right and left hyperideals of $R$ respectively. Clearly, $А Г B \subseteq A \cap B$. Since $R$ is regular, for $x \in R$ we have $x \in x \alpha a \beta x$ for some $a \in R$ and $\alpha, \beta \in \Gamma$. Now let $x \in A \cap B$. Then $x \alpha a \subseteq A$ and $x \in B$, thus $x \in x \alpha a \beta x \subseteq A$. B. Hence $А Г B=A \cap B$.

Conversely, let $x \in R$. Now $\langle x\rangle_{r}=\{w|w \in x \gamma r+n x| r \in R, n \in Z, \gamma \in \Gamma\}$ is a right hyperideal generated by $x$ and $\langle x\rangle_{l}=\{w|w \in r \gamma x+n x| r \in R, n \in$ $Z, \gamma \in \Gamma\}$ is a left hyperideal generated by $x$. Then $x \gamma 0+1 \cdot x=0 \gamma x+1 \cdot x=$ $x \in\langle x\rangle_{r} \cap\langle x\rangle_{l}=\langle x\rangle_{r} \Gamma\langle x\rangle_{l}$. Therefore $x \in x \Gamma R \Gamma x$ or $x \in n_{1} \cdot x^{3}, n_{1} \in Z$ where $x^{3}=x \Gamma x \Gamma x$. Hence $R$ is regular.

Theorem 2 Let $(\mathrm{R},+, \Gamma)$ be a $\Gamma$-hyperring. $\mathrm{R}$ is regular if and only if $\lambda \circ \mu=$ $\lambda \wedge \mu$ for any $\mathrm{T}$-fuzzy right hyperideal $\lambda$ and $\mathrm{T}$-fuzzy left hyperideal $\mu$ of $\mathrm{R}$. 
Proof. Let $\mathrm{R}$ be a regular $\Gamma$-hyperring. Let $\lambda, \mu$ be the $\mathrm{T}$-fuzzy right and left hyperideals of $\Gamma$-hyperring $R$ respectively. Let $x \in R, \gamma \in \Gamma$.

$$
\begin{aligned}
(\lambda \circ \mu)(x) & =\sup _{x \in y \gamma z} T(\lambda(y), \mu(z)) \leq \sup _{x \in y \gamma z} T(\lambda(x), \mu(x)) \\
& =(\lambda \wedge \mu)(x) .
\end{aligned}
$$

Thus $\lambda \circ \mu \subseteq \lambda \wedge \mu$. Since $R$ is regular, for $x \in R$ we have $x \in x \alpha a \beta x$ for some $a \in R, \alpha, \beta \in \Gamma$.

$$
\begin{aligned}
(\lambda \circ \mu)(x) & =\sup _{x \in y \gamma z} T(\lambda(y), \mu(z)) \\
& \geq T\left(\sup _{s \in x \alpha a} \lambda(s), \mu(x)\right) \\
& \geq T(\lambda(x), \mu(x)) \\
& =(\lambda \wedge \mu)(x) .
\end{aligned}
$$

Hence $\lambda \circ \mu=\lambda \wedge \mu$.

Conversely, let us assume that $\lambda \circ \mu=\lambda \wedge \mu$ for any T-fuzzy right hyperideal $\lambda$ and T-fuzzy left hyperideal $\mu$. Let $A, B$ be the right and left hyperideals of $\Gamma$-hyperring $R$ respectively. Then $\chi_{A}, \chi_{B}$ are the $T$-fuzzy right and left hyperideals of $\Gamma$-hyperring $R$ respectively. Clearly, АГB $\subseteq A \cap B$. Now $x \in A \cap B$. Then $\chi_{A}(x)=\chi_{B}(x)=1$. Thus $(\lambda \wedge \mu)(x)=T(\lambda(x), \mu(x))=1$. Therefore $(\lambda \circ \mu)(x)=1$. Then there is $a \in A, b \in B, \gamma \in \Gamma$ such that $x \in a \gamma b$. Thus $x \in A \Gamma B$. Hence $A \Gamma B=A \cap B$. Then by Lemma $2, R$ is regular.

Corollary 3 Let $(\mathrm{R},+, \Gamma)$ be a $\Gamma$-hyperring. $\mathrm{R}$ is regular if and only if $\lambda \circ \mu=$ $\lambda \cap \mu$ for any fuzzy right hyperideal $\lambda$ and fuzzy left hyperideal $\mu$ of $R$.

Proof. By taking min as t-norm $\mathrm{T}$ in Theorem 2, we get the required result.

Let $\left\{\mu_{i} \mid i \in \Lambda\right\}$ be a family of fuzzy sets in the $\Gamma$-hyperring $(R,+, \Gamma)$. We define the join $\bigvee_{i \in \Lambda} \mu_{i}$ and meet $\bigwedge_{i \in \Lambda} \mu_{i}$ as follows:

$$
\left(\bigvee_{i \in \Lambda} \mu_{i}\right)(x)=\sup \left\{\mu_{i}(x) \mid i \in \Lambda\right\},\left(\bigwedge_{i \in \Lambda} \mu_{i}\right)(x)=\inf \left\{\mu_{i}(x) \mid i \in \Lambda\right\}
$$

for all $x \in R$, where $\Lambda$ is any index set.

Theorem 3 Let $(\mathrm{R},+, \Gamma)$ be a $\Gamma$-hyperring. Then the family of $\mathrm{T}$-fuzzy hyperideals in $\mathrm{R}$ is a completely distributive lattice with respect to meet " $\wedge$ " and join " $\vee$ ". 
Proof. Since $[0,1]$ is a completely distributive lattice with respect to the usual ordering in $[0,1]$, it is sufficient to show that $\bigvee_{i \in \Lambda} \mu_{i}$ and $\bigwedge_{i \in \Lambda} \mu_{i}$ are T-fuzzy hyperideals of $R$ for a family of $T$-fuzzy hyperideals $\left\{\mu_{i} \mid i \in \Lambda\right\}$. For any $x, y \in R$, we have

$$
\begin{aligned}
\inf _{a \in x-y}\left(\bigvee_{i \in \Lambda} \mu_{i}\right)(a) & =\sup \left\{\inf _{a \in x-y} \mu_{i}(a) \mid i \in \Lambda\right\} \\
& \geq \sup \left\{T\left(\mu_{i}(x), \mu_{i}(y)\right) \mid i \in \Lambda\right\} \\
& \geq T\left(\sup \left\{\mu_{i}(x) \mid i \in \Lambda\right\}, \sup \left\{\mu_{i}(y) \mid i \in \Lambda\right\}\right) \\
& =T\left(\left(\bigvee_{i \in \Lambda} \mu_{i}\right)(x),\left(\bigvee_{i \in \Lambda} \mu_{i}\right)(y)\right) \\
\inf _{a \in x-y}\left(\bigwedge_{i \in \Lambda} \mu_{i}\right)(a) & =\inf \left\{\inf _{a \in x-y} \mu_{i a}(a) \mid i \in \Lambda\right\} \\
& \geq \inf \left\{T\left(\mu_{i}(x), \mu_{i}(y)\right) \mid i \in \Lambda\right\} \\
& \geq T\left(\inf \left\{\mu_{i}(x) \mid i \in \Lambda\right\}, \inf \left\{\mu_{i}(y) \mid i \in \Lambda\right\}\right) \\
& =T\left(\left(\bigwedge_{i \in \Lambda} \mu_{i}\right)(x),\left(\bigwedge_{i \in \Lambda} \mu_{i}\right)(y)\right) .
\end{aligned}
$$

Now let $x, y \in R, \gamma \in \Gamma$. Then

$$
\begin{aligned}
\inf _{a \in x \gamma y}\left(\bigvee_{i \in \Lambda} \mu_{i}\right)(a) & =\sup \left\{\inf _{a \in x \gamma y} \mu_{i}(a) \mid i \in \Lambda\right\} \\
& \geq \sup \left\{\mu_{i}(y) \mid i \in \Lambda\right\} \\
& =\left(\bigvee_{i \in \Lambda} \mu_{i}\right)(y), \\
\inf _{a \in x \gamma y}\left(\bigwedge_{i \in \Lambda} \mu_{i}\right)(a) & =\inf \left\{\inf _{a \in x \gamma y} \mu_{i}(a) \mid i \in \Lambda\right\} \\
& \geq \inf \left\{\mu_{i}(y) \mid i \in \Lambda\right\} \\
& =\left(\bigwedge_{i \in \Lambda} \mu_{i}\right)(y) .
\end{aligned}
$$

Hence $\bigvee_{i \in \Lambda} \mu_{i}$ and $\bigwedge_{i \in \Lambda} \mu_{i}$ are T-fuzzy hyperideals of $R$. 
Definition 15 Let $(\mathrm{R},+, \Gamma)$ be a $\Gamma$-hyperring and $\mathrm{T}$ be a $\mathrm{t}$-norm. A fuzzy set $\mu$ in $\mathrm{R}$ is said to satisfy imaginable property if $\operatorname{Im}(\mu) \subseteq \mathrm{E}_{\mathrm{T}}=\{\alpha \in[0,1] \mid$ $\mathrm{T}(\alpha, \alpha)=\alpha\}$.

Theorem 4 Let $(\mathrm{R},+, \Gamma)$ be a $\Gamma$-hyperring, $\mathrm{T}$ be a $\mathrm{t}$-norm and $\mu$ be an imaginable fuzzy set in $\mathrm{R}$. If each non-empty upper level set $\mathrm{U}(\mu ; \alpha)$ of $\mu$ is a hyperideal of $\mathrm{R}$, then $\mu$ is imaginable $\mathrm{T}$-fuzzy hyperideal of $\mathrm{R}$.

Proof. Let us suppose that each non-empty upper level set $U(\mu ; \alpha)$ of $\mu$ is a hyperideal of $R$. Then we first show that $\inf _{a \in x-y} \mu(a) \geq \min (\mu(x), \mu(y))$ for all $x, y \in R$. In fact, if not, then there exist $x_{0}, y_{0} \in R$ such that $\inf _{a \in x_{0}-y_{0}} \mu(a)<$ $\min \left(\mu\left(x_{0}\right), \mu\left(y_{0}\right)\right)$. Taking

$$
\alpha_{0}=\frac{1}{2}\left(\inf _{a \in x_{0}-y_{0}} \mu(a)+\min \left(\mu\left(x_{0}\right), \mu\left(y_{0}\right)\right)\right),
$$

we get $\inf _{a \in x_{0}-y_{0}} \mu(a)<\alpha_{0}<\min \left(\mu\left(x_{0}\right), \mu\left(y_{0}\right)\right)$ and thus $x_{0}, y_{0} \in U\left(\mu ; \alpha_{0}\right)$ and $x_{0}-y_{0} \nsubseteq \mathbb{U}\left(\mu ; \alpha_{0}\right)$. This is a contradiction. Hence

$$
\inf _{a \in x-y} \mu(a) \geq \min (\mu(x), \mu(y)) \geq T(\mu(x), \mu(y))
$$

for all $x, y \in R$. Now if the condition (2) of Definition 14 is not true, then $\inf _{b \in x_{0} \gamma y_{0}} \mu(b)<\mu\left(y_{0}\right)$ for some $x_{0}, y_{0} \in R, \gamma \in \Gamma$. Taking $s_{1}=\frac{1}{2}\left(\inf _{b \in x_{0} \gamma y_{0}} \mu(b)+\right.$ $\left.\mu\left(y_{0}\right)\right)$, then $0 \leq s_{1}<\mu\left(y_{0}\right)$ and $\inf _{b \in x_{0} \gamma y_{0}} \mu(b)<s_{1}$. Hence $y_{0} \in U\left(\mu ; s_{1}\right)$ and $x_{0} \gamma y_{0} \nsubseteq \mathbb{U}\left(\mu ; s_{1}\right)$, a contradiction. This completes the proof.

\section{T-fuzzy quasi-hyperideals and T-fuzzy bi-hyperideals in $\Gamma$-hyperrings}

In this section, we introduce the notions of fuzzy quasi(bi)-hyperideal and T-fuzzy quasi(bi)-hyperideal in $\Gamma$-hyperrings and some properties of them are studied. Also, the regular $\Gamma$-hyperrings are studied in terms of T-fuzzy quasihyperideals and T-fuzzy bi-hyperideals.

Definition 16 Let $(\mathrm{R},+, \Gamma)$ be a $\Gamma$-hyperring. A fuzzy subset $\mu$ of $\mathrm{R}$ is called T-fuzzy quasi-hyperideal if

1. $\inf _{a \in x-y} \mu(a) \geq T(\mu(x), \mu(y))$, 
2. $\left(\mu \circ \chi_{R}\right) \wedge\left(\chi_{R} \circ \mu\right) \subseteq \mu$ for all $x, y \in R$.

If $\mathrm{T}=\min$, then $\mu$ is called fuzzy quasi-hyperideal of $\mathrm{R}$.

Lemma 3 Let $(\mathrm{R},+, \cdot)$ be a hyperring. A fuzzy subset $\mu$ is a $\mathrm{T}$-fuzzy quasihyperideal of $\mathrm{R}$ if and only if

(a) $\mu(x) \geq T\left[\sup _{x \in y \gamma z} \mu(y), \sup _{x \in y \gamma z} \mu(z)\right], \forall x \in R, \gamma \in \Gamma$ and

(b) $\inf _{a \in x-y} \mu(a) \geq T(\mu(x), \mu(y)), \forall x, y \in R$.

Proof. Let $\mu$ be a T-fuzzy quasi-hyperideal of R. Let $x \in R$. Then

$$
\begin{aligned}
\mu(x) & \geq T\left(\left(\mu \circ \chi_{R}\right)(x),\left(\chi_{R} \circ \mu(x)\right)\right) \\
& =T\left(\sup _{x \in y \gamma z} \mathrm{~T}\left(\mu(y), \chi_{R}(z)\right), \sup _{x \in y \gamma z} \mathrm{~T}\left(\left(\chi_{R}\right)(y), \mu(z)\right)\right. \\
& =\mathrm{T}\left(\sup _{x \in y \gamma z} \mu(y), \sup _{x \in y \gamma z} \mu(z)\right) .
\end{aligned}
$$

Conversely,

$$
\begin{aligned}
\mu(x) & \geq \mathrm{T}\left(\sup _{x \in y \gamma z} \mu(y), \sup _{x \in y \gamma z} \mu(z)\right) \\
& =\mathrm{T}\left(\sup _{x \in y \gamma z} \mathrm{~T}\left(\mu(y), \chi_{R}(z)\right), \sup _{x \in y \gamma z} \mathrm{~T}\left(\left(\chi_{R}\right)(y), \mu(z)\right)\right) \\
& =\mathrm{T}\left(\left(\mu \circ \chi_{R}\right)(x),\left(\chi_{R} \circ \mu\right)(x)\right) \\
& =\left(\left(\mu \circ \chi_{R}\right) \wedge\left(\chi_{R} \circ \mu\right)\right)(x) .
\end{aligned}
$$

Then $\mu$ is a T-fuzzy quasi-hyperideal of $R$.

Corollary 4 Let $(\mathrm{R},+, \cdot)$ be a $\Gamma$-hyperring. A fuzzy subset $\mu$ is a fuzzy quasihyperideal of $\mathrm{R}$ if and only if

1. $\mu(x) \geq \min \left\{\sup _{x \in y \gamma z} \mu(y), \sup _{x \in y \gamma z} \mu(z)\right\}, \forall x \in R, \gamma \in \Gamma$ and

2. $\inf _{a \in x-y} \mu(a) \geq \min \{\mu(x), \mu(y)\}, \forall x, y \in R$.

Proof. By taking min as $\mathrm{t}$-norm $\mathrm{T}$ in Lemma 3, we get the required result. 
Lemma 4 Let $(\mathrm{R},+, \Gamma)$ be a $\Gamma$-hyperring. Every fuzzy quasi-hyperideal of $\mathrm{R}$ is a T-fuzzy quasi-hyperideal of $\mathrm{R}$.

Proof. Let $\mu$ be a fuzzy quasi-hyperideal of R. Let $x, y \in R$. Then

$$
\inf _{a \in x-y} \mu(a) \geq \min \{\mu(x), \mu(y)\} \geq T(\mu(x), \mu(y))
$$

and

$$
\mu(x) \geq \min \left\{\sup _{x \in y \gamma z} \mu(y), \sup _{x \in y \gamma z} \mu(z)\right\} \geq T\left[\sup _{x \in y \gamma z} \mu(y), \sup _{x \in y \gamma z} \mu(z)\right] .
$$

Thus $\mu$ is a T-fuzzy quasi-hyperideal of $R$.

Corollary 5 Let $(\mathrm{R},+, \Gamma)$ be a $\Gamma$-hyperring. If $\mathrm{Q}$ is a quasi-hyperideal in $\mathrm{R}$, then $\mathrm{\chi}_{\mathrm{Q}}$ is a $\mathrm{T}$-fuzzy quasi-hyperideal of $\mathrm{R}$.

Theorem 5 Let $(\mathrm{R},+, \Gamma)$ be a $\Gamma$-hyperring. Every $\mathrm{T}$-fuzzy right hyperideal of $\mathrm{R}$ is $\mathrm{T}$-fuzzy quasi-hyperideal. Moreover, every $\mathrm{T}$-fuzzy left hyperideal of $\mathrm{R}$ is T-fuzzy quasi-hyperideal.

Proof. Let $\mu$ be a T-fuzzy right hyperideal of R. Let $x \in R$. Then $\inf _{a \in y \gamma z} \mu(a) \geq$ $\mu(y)$. If $x \in y \gamma z$, then

$$
\begin{aligned}
\mu(x) & \geq \mu(y) \geq \min \left\{\sup _{x \in y \gamma z} \mu(y), \sup _{x \in y \gamma z} \mu(z)\right\} \\
& \geq T\left[\sup _{x \in y \gamma z} \mu(y), \sup _{x \in y \gamma z} \mu(z)\right] .
\end{aligned}
$$

Therefore $\mu$ is a T-fuzzy quasi-hyperideal. Similarly, if $\lambda$ is a T-fuzzy left hyperideal of $R$, then $\lambda$ is a T-fuzzy quasi-hyperideal.

Theorem 6 Let $(\mathrm{R},+, \Gamma)$ be a $\Gamma$-hyperring. If $\mu, \lambda$ are T-fuzzy quasi-hyperideals of $\mathrm{R}$, then $\mu \wedge \lambda$ is a $\mathrm{T}$-fuzzy quasi-hyperideal of $\mathrm{R}$.

Proof. Let $\mu, \lambda$ be the T-fuzzy quasi-hyperideal of R. Let $x, y \in R$. From the proof of the Theorem 1, we have $\inf _{a \in x-y}(\mu \wedge \lambda)(a) \geq T((\mu \wedge \lambda)(x),(\mu \wedge \lambda)(y))$.

We have

$$
(\mu \wedge \lambda)(x)=T(\mu(x), \lambda(x))
$$




$$
\begin{aligned}
& \geq \mathrm{T}\left(\mathrm{T}\left(\sup _{x \in y \gamma z} \mu(y), \sup _{x \in y \gamma z} \mu(z)\right), \mathrm{T}\left(\sup _{x \in y \gamma z} \lambda(y), \sup _{x \in y \gamma z} \lambda(z)\right)\right) \\
& =\mathrm{T}\left(\mathrm{T}\left(\mathrm{T}\left(\sup _{x \in y \gamma z} \mu(y), \sup _{x \in y \gamma z} \mu(z)\right), \sup _{x \in y \gamma z} \lambda(y)\right), \sup _{x \in y \gamma z} \lambda(z)\right) \\
& =\mathrm{T}\left(\mathrm{T}\left(\mathrm{T}\left(\sup _{x \in y \gamma z} \mu(y), \sup _{x \in y \gamma z} \lambda(y)\right), \sup _{x \in y \gamma z} \mu(z)\right), \sup _{x \in y \gamma z} \lambda(z)\right) \\
& =\mathrm{T}\left(\mathrm{T}\left(\sup _{x \in y \gamma z} \mu(y), \sup _{x \in y \gamma z} \lambda(y)\right), \mathrm{T}\left(\sup _{x \in y \gamma z} \mu(z), \sup _{x \in y \gamma z} \lambda(z)\right)\right) \\
& \geq \mathrm{T}(\mathrm{T}(\mu(y), \lambda(y)), \mathrm{T}(\mu(z), \lambda(z))) \\
& \geq \mathrm{T}\left(\sup _{x \in y \gamma z} \mathrm{~T}(\mu(y), \lambda(y)), \sup _{x \in y \gamma z} \mathrm{~T}(\mu(z), \lambda(z))\right) \\
& =\mathrm{T}\left(\sup _{x \in y \gamma z}(\mu \wedge \lambda)(y), \sup _{x \in y \gamma z}(\mu \wedge \lambda)(z)\right) .
\end{aligned}
$$

Therefore $\mu \wedge \lambda$ is a T-fuzzy quasi-hyperideal of $R$.

Theorem 7 Let $(\mathrm{R},+, \Gamma)$ be a $\Gamma$-hyperring. If $\mu, \lambda$ are $\mathrm{T}$-fuzzy right and $\mathrm{T}$-fuzzy left hyperideals of $\mathrm{R}$ respectively, then $\mu \wedge \lambda$ is a $\mathrm{T}$-fuzzy quasi-hyperideal of $R$.

Proof. Let $\mu$ and $\lambda$ be T-fuzzy right and T-fuzzy left hyperideal of $R$ respectively. Then by Theorem 5, $\mu$ and $\lambda$ are T-fuzzy quasi-hyperideals of R. By Theorem $6, \mu \wedge \lambda$ is a T-fuzzy quasi-hyperideal of $R$.

Definition 17 Let $(\mathrm{R},+, \Gamma)$ be a $\Gamma$-hyperring. A fuzzy subset $\mu$ of $\mathrm{R}$ is called T-fuzzy bi-hyperideal if

1. $\inf _{a \in x-y} \mu(a) \geq T(\mu(x), \mu(y))$,

2. $\inf _{a \in x \gamma_{1} y \gamma_{2} z} \mu(a) \geq T(\mu(x), \mu(z))$ for all $x, y, z \in R, \gamma_{1}, \gamma_{2} \in \Gamma$.

If $\mathrm{T}=\min$, then $\mu$ is called fuzzy bi-hyperideal of $\mathrm{R}$.

Lemma 5 Let $\mathrm{B}$ be a bi-hyperideal of a $\Gamma$-hyperring $\mathrm{R}$. For any $0<\alpha<1$, there exists a fuzzy bi-hyperideal $\mu$ such that $\mathrm{U}(\mu ; \alpha)=\mathrm{B}$.

Proof. Let B be a bi-hyperideal of $R$. Define $\mu: R \rightarrow[0,1]$ by

$$
\mu(x)= \begin{cases}\alpha, & \text { if } x \in B \\ 0, & \text { if } x \notin B\end{cases}
$$


where $\alpha$ is a fixed number in $(0,1)$. It is clear that $U(\mu ; \alpha)=B$. Let $x, y \in R$. If $x, y \in B$, then $\inf _{a \in x-y} \mu(a)=\alpha=\min \{\mu(x), \mu(y)\}$. If at least one of $x$ and $y$ is not in $B$, then $\inf _{a \in x-y} \mu(a)=0=\min \{\mu(x), \mu(y)\}$. Let $x, y, z \in R$. If $x, z \in B$, then $\mu(x)=\alpha, \mu(z)=\alpha$. Also $\inf _{a \in x \gamma y \cdot z} \mu(a)=\alpha \geq \min \{\mu(x), \mu(z)\}$. If at least one of $x$ and $z$ is not in $B$, then $\inf _{a \in x \gamma y \cdot z} \mu(a)=0=\min \{\mu(x), \mu(z)\}$. Thus $\mu$ is a fuzzy bi-hyperideal of $R$.

Lemma 6 Let $\mathrm{B}$ be a non-empty subset of a $\Gamma$-hyperring $\mathrm{R}$. B is a bi-hyperideal of $\mathrm{R}$ if and only if $\mathrm{X}_{\mathrm{B}}$ is a fuzzy bi-hyperideal of $\mathrm{R}$.

Proof. (i) Let $x, y \in R$.

Case 1: $x, y \in B$. Then $\chi_{B}(x)=\chi_{B}(y)=1$. Therefore $\inf _{a \in x-y} \chi_{B}(a)=1 \geq$ $\min \left\{\chi_{B}(x), \chi_{B}(y)\right\}$.

Case 2: $x \in B$ and $y \notin B$. Then $\chi_{B}(x)=1$ and $x_{B}(y)=0$. Therefore $\inf _{a \in x-y} \chi_{B}(a)=0 \geq \min \left\{\chi_{B}(x), \chi_{B}(y)\right\}$.

Case 3: $x \notin B$ and $y \in B$. Then $\chi_{B}(x)=0$ and $\chi_{B}(y)=1$. Therefore $\inf _{a \in x-y} \chi_{B}(a)=0 \geq \min \left\{\chi_{B}(x), \chi_{B}(y)\right\}$.

Case 4: $x \notin B$ and $y \notin B$. Then $\chi_{B}(x)=0$ and $\chi_{B}(y)=0$. Therefore $\inf _{a \in x-y} \chi_{B}(a)=0 \geq \min \left\{\chi_{B}(x), \chi_{B}(y)\right\}$.

(ii) Let $x, y, z \in R, \gamma_{1}, \gamma_{2} \in \Gamma$.

Case 1: $x, z \in B$. Then $\chi_{B}(x)=\chi_{B}(z)=1$. Therefore $\inf _{a \in x \gamma_{1} y \gamma_{2} z} \chi_{B}(a)=1 \geq$ $\min \left\{\chi_{\mathrm{B}}(\mathrm{x}), \chi_{\mathrm{B}}(z)\right\}$.

Case 2: $x \in B$ and $z \notin B$. Then $\chi_{B}(x)=1$ and $\chi_{B}(z)=0$. Therefore $\inf _{a \in x \gamma_{1} y \gamma_{2} z} \chi_{B}(a)=0 \geq \min \left\{\chi_{B}(x), \chi_{B}(z)\right\}$.

Case 3: $x \notin B$ and $z \in B$. Then $\chi_{B}(x)=0$ and $\chi_{B}(z)=1$. Therefore $\inf _{a \in x \gamma_{1} y \gamma_{2} z} \chi_{B}(a)=0 \geq \min \left\{\chi_{B}(x), \chi_{B}(z)\right\}$.

Case 4: $x \notin B$ and $z \notin B$. Then $\chi_{B}(x)=0$ and $\chi_{B}(z)=0$. Therefore $\inf _{a \in x \gamma_{1} y \gamma_{2} z} \chi_{B}(a)=0 \geq \min \left\{\chi_{B}(x), \chi_{B}(z)\right\}$.

Thus $\chi_{B}$ is a fuzzy bi-hyperideal of $R$. Conversely, let us suppose that $\chi_{B}$ is a fuzzy bi-hyperideal of R. Then, by Lemma $5, \chi_{B}$ is two-valued. Hence B is a bi-hyperideal of $\mathrm{R}$.

Lemma 7 Let $(\mathrm{R},+, \Gamma)$ be a $\Gamma$-hyperring. A fuzzy subset $\mu$ is a $\mathrm{T}$-fuzzy bihyperideal of $\mathrm{R}$ if and only if $\mu \circ \chi_{R} \circ \mu \subseteq \mu$. 
Proof. Let $\mu$ be a T-fuzzy bi-hyperideal of R. Let $x \in R, \gamma_{1}, \gamma_{2}$. If $x \notin a \gamma_{1} b \gamma_{2} c$ for $a, b, c \in R$, then $\mu(x) \geq\left(\mu \circ \chi_{R} \circ \mu\right)(x)=0$. If $x \in a \gamma_{1} b \gamma_{2} c$, then

$$
\begin{aligned}
\mu(x) & \geq T(\mu(a), \mu(c))=T(\mu(a), T(1, \mu(c))) \\
& =T\left(\mu(a), T\left(\chi_{R}(b), \mu(c)\right)\right) .
\end{aligned}
$$

Therefore,

$$
\mu(x) \geq \sup _{x \in a \gamma_{1} y} T\left(\mu(a), \sup _{y \in b \gamma_{2} c} T\left(\chi_{R}(b), \mu(c)\right)\right) \geq\left(\mu \circ \chi_{R} \circ \mu\right)(x) .
$$

Conversely, it is clear that $\inf _{a \in x \alpha y \beta z} \mu(a) \geq T(\mu(x), \mu(z))$.

Theorem 8 Let $(\mathrm{R},+, \Gamma)$ be a $\Gamma$-hyperring. Every $\mathrm{T}$-fuzzy quasi-hyperideal of $\mathrm{R}$ is a $\mathrm{T}$-fuzzy bi-hyperideal of $\mathrm{R}$.

Proof. Let $\mu$ be a T-fuzzy quasi-hyperideal of R. Let $x, y, z \in R, \alpha, \beta$. Then $\inf _{a \in x \alpha y \beta z} \mu(a) \geq T\left(\mu(x), \inf _{s \in y \beta z} \mu(s)\right) \geq T(\mu(x), \mu(z))$. Therefore $\mu$ is a T-fuzzy bi-hyperideal of $\mathrm{R}$.

Theorem 9 Let $(\mathrm{R},+, \Gamma)$ be a $\Gamma$-hyperring. Every fuzzy bi-hyperideal of $\mathrm{R}$ is $a \mathrm{~T}$-fuzzy bi-hyperideal of $\mathrm{R}$.

Proof. Let $\mu$ be a fuzzy bi-hyperideal. Let $x, y, z \in R, \alpha, \beta \in \Gamma$. Then

$$
\inf _{a \in x-y} \mu(a) \geq \min \{\mu(x), \mu(y)\} \geq T(\mu(x), \mu(y))
$$

and

$$
\inf _{a \in x \alpha y \beta z} \mu(a) \geq \min \{\mu(x), \mu(z)\} \geq T(\mu(x), \mu(z)) .
$$

Thus $\mu$ is a T-fuzzy bi-hyperideal of $R$.

Corollary 6 Let $(\mathrm{R},+, \Gamma)$ be a $\Gamma$-hyperring. If $\mathrm{B}$ is a bi-hyperideal in $\mathrm{R}$, then $\chi_{\mathrm{B}}$ is a $\mathrm{T}$-fuzzy bi-hyperideal of $\mathrm{R}$.

Theorem 10 Let $(\mathrm{R},+, \Gamma)$ be a $\Gamma$-hyperring. If $\mu$ and $\lambda$ are $\mathrm{T}$-fuzzy bi-hyperideals of $\mathrm{R}$, then $\mu \wedge \lambda$ is a $\mathrm{T}$-fuzzy bi-hyperideal of $\mathrm{R}$. 
Proof. Let $\mu$ and $\lambda$ be T-fuzzy bi-hyperideals of R. Then

$$
\begin{aligned}
\inf _{\mathrm{a} \in x \alpha y \beta z}(\mu \wedge \lambda)(a) & \geq \mathrm{T}(\mathrm{T}(\mu(x), \mu(z)), \mathrm{T}(\lambda(x), \lambda(z))) \\
& =\mathrm{T}(\mathrm{T}(\mu(x), \lambda(x)), \mathrm{T}(\mu(x), \lambda(z))) \\
& =\mathrm{T}((\mu \wedge \lambda)(x),(\mu \wedge \lambda)(z)) .
\end{aligned}
$$

Hence $\mu \wedge \lambda$ is a T-fuzzy bi-hyperideal of $R$.

Corollary 7 Let $(\mathrm{R},+, \Gamma)$ be a $\Gamma$-hyperring. If $\mu$ and $\lambda$ are fuzzy bi-hyperideals of $\mathrm{R}$, then $\mu \cap \lambda$ is a fuzzy bi-hyperideal of $\mathrm{R}$.

Lemma 8 Let $(\mathrm{R},+, \cdot)$ be a $\Gamma$-hyperring. Then the following statements are equivalent:

1. $\mathrm{R}$ is regular.

2. $\mathrm{B}=\mathrm{B} Г \mathrm{R} Г \mathrm{~B}$, for any bi-hyperideal $\mathrm{B}$ in $\mathrm{R}$.

3. $\mathrm{Q}=\mathrm{Q} \Gamma \mathrm{R} \Gamma \mathrm{Q}$ for any quasi-hyperideal $\mathrm{Q}$ in $\mathrm{R}$.

Proof. $(1) \Rightarrow(2)$. Let us assume that $R$ is a regular $\Gamma$-hyperring. Let $B$ be a bi-hyperideal of $R$ and $a \in B$. Then there exist $x \in R, \alpha, \beta \in \Gamma$ such that $a \in a \alpha x \beta a \subseteq B \Gamma R \Gamma B$. Hence, $B \subseteq$ ВГRГB. Since $B$ is a bi-hyperideal, then $\mathrm{B} Г \mathrm{R} Г \mathrm{~B} \subseteq \mathrm{B}$. Therefore $\mathrm{B}=\mathrm{B} Г \mathrm{R} Г \mathrm{~B}$.

$(2) \Rightarrow(3)$. Let $\mathrm{Q}$ be a quasi-hyperideal of $\mathrm{R}$, that is $\mathrm{Q} \Gamma \mathrm{R} \cap \mathrm{R} \Gamma \mathrm{Q} \subseteq \mathrm{Q}$. Since every quasi-hyperideal is a bi-hyperideal of $R$, then we have $Q \Gamma R \Gamma Q=Q$.

(3) $\Rightarrow(1)$. Let us assume that (3) holds. Let I and J be any right hyperideal and left hyperideal of $R$, respectively. Then we have $(I \cap J) \Gamma R \cap R \Gamma(I \cap J) \subseteq I \cap J$. It is easy to see that $I \cap J$ is a quasi-hyperideal of $R$. By (3) and Lemma 2, we have $\mathrm{I} \cap \mathrm{J} \subseteq(\mathrm{I} \cap \mathrm{J}) \Gamma \mathrm{R} \Gamma(\mathrm{I} \cap \mathrm{J}) \subseteq \mathrm{I} \Gamma \mathrm{R} \Gamma \subseteq \mathrm{I} \Gamma \mathrm{J} \subseteq \mathrm{I} \cap \mathrm{J}$. Hence, $\mathrm{I} \Gamma \mathrm{J}=\mathrm{I} \cap \mathrm{J}$ and so $R$ is regular.

Theorem 11 Let $(\mathrm{R},+, \Gamma)$ be a $\Gamma$-hyperring. Then the following statements are equivalent:

1. $\mathrm{R}$ is regular.

2. $\lambda \wedge \lambda \subseteq \lambda \circ \chi_{R} \circ \lambda \subseteq \lambda$ for any $\mathrm{T}$-fuzzy bi-hyperideal $\lambda$ of $\mathrm{R}$.

3. $\mu \wedge \mu \subseteq \mu \circ \chi_{R} \circ \mu \subseteq \mu$ for any $\mathrm{T}$-fuzzy quasi-hyperideal $\mu$ of $\mathrm{R}$. 
Proof. $(1) \Rightarrow(2)$. Let $(R,+, \Gamma)$ be a regular $\Gamma$-hyperring and let $\lambda$ be a $T$-fuzzy bi-hyperideal of R. Let $x \in R$. By Lemma $7, \lambda(x) \geq\left(\lambda \circ \chi_{R} \circ \lambda\right)(x)$. For $x \in R$ there is $a \in R, \alpha, \beta \in \Gamma$ such that $x \in x \alpha a \beta x$. Thus $\lambda(x) \geq \inf _{s \in x \alpha a \beta x} \lambda(s) \geq$ $\mathrm{T}(\lambda(x), \lambda(x))=(\lambda \wedge \lambda)(x)$. Hence $\lambda \wedge \lambda \subseteq \lambda \circ \chi_{R} \circ \lambda \subseteq \lambda$.

$(2) \Rightarrow(3)$. It is trivial.

$(3) \Rightarrow(1)$. Let $\mathrm{Q}$ be any quasi-hyperideal in $\mathrm{R}$. Then by Corollary $5, \mathrm{\chi}_{\mathrm{Q}}$ is a T-fuzzy quasi-hyperideal. It is clear that $\mathrm{Q} \Gamma \mathrm{R} \Gamma \mathrm{Q} \subseteq \mathrm{Q}$. Let $x \in \mathrm{Q}$. Then $\chi_{Q}(x)=1$. Thus $\left(\chi_{Q} \circ \chi_{R} \circ \chi_{Q}\right)(x) \geq T\left(\chi_{Q}(x), \chi_{Q}(x)\right)=1$. Then $\left(\chi_{Q} \circ \chi_{R} \circ\right.$ $\left.\chi_{\mathrm{Q}}\right)(\mathrm{x})=1$. Thus $\mathrm{x} \in \mathrm{Q} \Gamma \mathrm{R} \Gamma \mathrm{Q}$. Therefore $\mathrm{Q} \subseteq \mathrm{Q} \Gamma \mathrm{R} \Gamma \mathrm{Q}$. Hence $\mathrm{Q}=\mathrm{Q} \Gamma \mathrm{R} \Gamma \mathrm{Q}$. By Lemma $8, \mathrm{R}$ is regular.

Corollary 8 Let $(\mathrm{R},+, \Gamma)$ be a $\Gamma$-hyperring. Then the following statements are equivalent:

1. $\mathrm{R}$ is regular.

2. $\lambda=\lambda \circ \chi_{R} \circ \lambda$ for any fuzzy bi-hyperideal $\lambda$ of $R$.

3. $\mu=\mu \circ \chi_{R} \circ \mu$ for any fuzzy quasi-hyperideal $\mu$ of $R$.

Proof. By taking min as t-norm $\mathrm{T}$ in Theorem 11, we get the required result.

Theorem 12 Let $(\mathrm{R},+, \Gamma)$ be a regular $\Gamma$-hyperring. If $\mu$ is an imaginable $\mathrm{T}$-fuzzy bi-hyperideal of $\mathrm{R}$, then $\mu$ is a $\mathrm{T}$-fuzzy quasi-hyperideal of $\mathrm{R}$.

Proof. Let $\mu$ be an imaginable T-fuzzy bi-hyperideal of a regular $\Gamma$-hyperring $R$ and $a \in R$. Suppose that $\left(\mu \circ \chi_{R}\right)(a) \leq \mu(a)$. Then we get:

$$
\begin{aligned}
\mu(a) & \geq\left(\mu \circ \chi_{R}\right)(a) \geq \min \left\{\left(\chi_{R} \circ \mu\right)(a),\left(\mu \circ \chi_{R}\right)(a)\right\} \\
& \geq T\left(\left(\chi_{R} \circ \mu\right)(a),\left(\mu \circ \chi_{R}\right)(a)\right) \\
& =\left(\chi_{R} \circ \mu \wedge \mu \circ \chi_{R}\right)(a)
\end{aligned}
$$

Suppose that $\left(\mu \circ \chi_{R}\right)(a)>\mu(a)$. Then $\sup _{a \in \gamma y} T\left(\mu(x), \chi_{R}(y)\right)>\mu(a)$. Thus $\mathrm{T}\left(\mu(x), \chi_{R}(y)\right)>\mu(a)$. So $\mu(x)>\mu(a)$.

Since $R$ is a regular $\Gamma$-hyperring, then there exists $b \in R, \alpha, \beta \in \Gamma$, such that $a \in a \alpha b \beta a \subseteq x \gamma y \alpha b \beta a$. Since $\mu$ is an imaginable T-fuzzy bi-hyperideal, then $\mu(a) \geq T(\mu(x), \mu(a))$. Then $T(\mu(a), \mu(a)) \geq T(\mu(x), \mu(a))$. This is a contradiction to $\mu(x)>\mu(a)$. Thus $\left(\mu \circ \chi_{R}\right)(x) \leq \mu(a)$.

In similar way, we can prove $\left(\chi_{R} \circ \mu\right)(a) \leq \mu(a)$. So $\mu \circ \chi_{R} \wedge \chi_{R} \circ \mu \subseteq \mu$. Hence $\mu$ is a T-fuzzy quasi-hyperideal of $R$. 


\section{On T- $(\lambda, \mu)$-fuzzy bi-hyperideals}

Definition 18 Let $(\mathrm{R},+, \Gamma)$ be a $\Gamma$-hyperring and $\mathrm{A}$ be a fuzzy subset of $\mathrm{R}$. Then $\mathrm{A}$ is called $a \mathrm{~T}-(\lambda, \mu)$-fuzzy bi-hyperideal of $\mathrm{R}$ if for all $x, y, z \in \mathrm{R}, \alpha, \beta \in \Gamma$, we have

$$
\begin{aligned}
& \text { 1. } \inf _{a \in x-y} A(a) \vee \lambda \geq T(T(A(x), A(y)), \mu) \\
& \text { 2. } \inf _{a \in x \propto y \beta z} A(a) \vee \lambda \geq T(T(A(x), A(z)), \mu)
\end{aligned}
$$

Theorem 13 Let $(\mathrm{R},+, \Gamma)$ be a $\Gamma$-hyperring and $\mathrm{A}$ be a fuzzy subset of $\mathrm{R}$. Then $\mathrm{A}$ is a $\mathrm{T}-(\lambda, \mu)$-fuzzy bi-hyperideal of $\mathrm{R}$ iff $\mathrm{A}_{\alpha}$ is a bi-hyperideal of $\mathrm{R}$ for all $\alpha \in(\lambda, \mu)$

Proof. Let $A$ be a T- $(\lambda, \mu)$-fuzzy bi-hyperideal of R. Let $x, y \in A_{\alpha}$. Then $A(x) \geq \alpha, A(y) \geq \alpha$. Consider $\inf _{a \in x-y} A(a) \vee \lambda \geq T(T(A(x), A(y)), \mu) \geq$ $\mathrm{T}(\mathrm{T}(\alpha, \alpha), \mu)=\alpha$ (since $\alpha>\lambda)$. That is, $\inf _{\mathrm{a} \in \mathrm{x}-\mathrm{y}} A(\mathrm{a}) \vee \lambda \geq \alpha$. This implies that $\inf _{a \in x-y} A(a) \geq \alpha$, that is, $x-y \subseteq A_{\alpha}$. Hence $A_{\alpha}$ is a subhypergroup of R. Let $x, z \in A_{\alpha}, y \in R$ and $\gamma, \beta \in \Gamma$. This implies that $A(x) \geq \alpha, A(z) \geq \alpha$. Then $\inf _{a \in x-y} A(a) \vee \lambda \geq T(T(A(x), A(z)), \mu) \geq T(T(\alpha, \alpha), \mu)=\alpha($ since $\alpha>\lambda)$. That is, $\inf _{a \in x \gamma y \beta z} A(a) \vee \lambda \geq \alpha$. This implies that $\inf _{a \in x \gamma y \beta z} A(a) \geq \alpha$ and so $x \gamma y \beta z \subseteq A_{\alpha}$. Hence $A_{\alpha}$ is a bi-hyperideal of $R$.

Conversely, let us suppose that $A_{\alpha}$ is a bi-hyperideal of $R$ for all $\alpha \in(\lambda, \mu)$. Suppose that $\inf _{a \in x-y} A(a) \vee \lambda<T(T(A(x), A(y)), \mu)=\alpha$. Then $\inf _{a \in x-y} A(a) \vee \lambda<$ $\alpha$ which implies that $\inf _{a \in x-y} A(a)<\alpha$ (since $\left.\alpha>\lambda\right)$. This implies that $x-y \not$ $A_{\alpha}$ which is impossible since $A_{\alpha}$ is a bi-hyperideal of R. Hence $\inf _{a \in x-y} A(a) \vee$ $\lambda \geq T(T(A(x), A(y)), \mu)$. Similarly, we can prove that $\inf _{a \in x \alpha y \beta z} A(a) \vee \lambda \geq$ $\mathrm{T}(\mathrm{T}(\mathrm{A}(\mathrm{x}), A(z)), \mu)$. Hence $A$ is a $\mathrm{T}-(\lambda, \mu)$-fuzzy bi-hyperideal of $\mathrm{R}$.

Definition 19 Let $\mathrm{R}$ and $\mathrm{R}^{\prime}$ be $\Gamma$ and $\Gamma^{\prime}$-hyperrings, respectively, $\varphi: R \rightarrow \mathrm{R}^{\prime}$ and $\mathrm{f}: \Gamma \rightarrow \Gamma^{\prime}$ be two maps. Then, $(\varphi, \mathrm{f})$ is called $a\left(\Gamma, \Gamma^{\prime}\right)$-homomorphism if

1. $\forall(x, y) \in R^{2}, \varphi(x+y)=\{\varphi(z) \mid z \in x+y\} \subseteq \varphi(x)+\varphi(y)$,

2. $\forall(x, y, \alpha) \in R^{2} \times \Gamma, \varphi(x \alpha y)=\{\varphi(z) \mid z \in x \alpha y\} \subseteq \varphi(x) f(\alpha) \varphi(y)$.

Let $\varphi$ be a mapping from a set $X$ to a set $Y$. Let $\mu$ be a fuzzy subset of $X$ and $\lambda$ be a fuzzy subset of $Y$. Then the inverse image $\varphi^{-1}(\lambda)$ of $\lambda$ is the fuzzy 
subset of $X$ defined by $\varphi^{-1}(\lambda)(x)=\lambda(\varphi(x))$ for all $x \in X$. The image $\varphi(\mu)$ of $\mu$ is the fuzzy subset of $Y$ defined by

$$
\varphi(\mu)(y)= \begin{cases}\sup \left\{\mu(t) \mid t \in \varphi^{-1}(y)\right\}, & \text { if } \varphi^{-1}(y) \neq \emptyset \\ 0, & \text { otherwise. }\end{cases}
$$

for all $y \in Y$.

Theorem 14 Let $(\varphi, f)$ be a onto $\left(\Gamma, \Gamma^{\prime}\right)$-homomorphism of $(R,+, \Gamma)$ and $\left(\mathrm{R}^{\prime},+, \Gamma^{\prime}\right)$ respectively and let $\mathrm{A}$ be a $\mathrm{T}-(\lambda, \mu)$-fuzzy bi-hyperideal of $\mathrm{R}$. Then $\varphi(\mathrm{A})$ is a $\mathrm{T}-(\lambda, \mu)$-fuzzy bi-hyperideal of $\mathrm{R}^{\prime}$.

Proof. Let $y_{1}, y_{2} \in R^{\prime}$. Then we have

$$
\begin{aligned}
& \inf _{z \in y_{1}-y_{2}}\{\varphi(A)(z)\} \vee \lambda=\sup _{z \in y_{1}-y_{2}}\left\{\inf _{a \in \varphi^{-1}\left(y_{1}\right)-\varphi^{-1}\left(y_{2}\right)} A(a)\right\} \vee \lambda \\
& =\sup _{z \in y_{1}-y_{2}}\left\{\inf _{a \in \varphi^{-1}\left(y_{1}\right)-\varphi^{-1}\left(y_{2}\right)} A(a) \vee \lambda\right\} \\
& \geq \sup _{\varphi(z) \in \varphi\left(y_{1}\right)-\varphi\left(y_{2}\right)}\{A((\varphi)(z))\} \vee \lambda \\
& \geq \sup \left\{\mathrm{T}\left(\mathrm{T}\left(\mathrm{A}\left((\varphi)\left(\mathrm{x}_{1}\right)\right), \mathrm{A}\left((\varphi)\left(\mathrm{x}_{2}\right)\right)\right), \mu\right) \mid\right. \\
& \left.x_{1} \in(\varphi)^{-1}\left(y_{1}\right), x_{2} \in(\varphi)^{-1}\left(y_{2}\right)\right\} \\
& \geq \mathrm{T}\left(\mathrm{T}\left(\sup \left\{\mathrm{A}\left((\varphi)\left(\mathrm{x}_{1}\right)\right)\right\}, \sup \left\{\mathrm{A}\left((\varphi)\left(x_{2}\right)\right)\right\}\right), \mu\right) \mid \\
& \left.x_{1} \in(\varphi)^{-1}\left(y_{1}\right), x_{2} \in(\varphi)^{-1}\left(y_{2}\right)\right\} \\
& =\mathrm{T}\left(\mathrm{T}\left((\varphi(A))\left(\mathrm{y}_{1}\right),(\varphi(A))\left(\mathrm{y}_{2}\right)\right), \mu\right)
\end{aligned}
$$

Therefore, $\inf _{z \in y_{1}-y_{2}}\{\varphi(A)(z)\} \vee \lambda \geq T\left(T\left((\varphi(A))\left(y_{1}\right),(\varphi(A))\left(y_{2}\right)\right), \mu\right)$.

Similarly, we can prove that

$$
\inf _{a \in y_{1} \alpha y_{2} \beta y_{3}}(\varphi(A))(a) \vee \lambda \geq T\left(T\left((\varphi(A))\left(y_{1}\right),(\varphi(A))\left(y_{3}\right)\right), \mu\right) .
$$

Hence $\varphi(A)$ is a $T-(\lambda, \mu)$-fuzzy bi-hyperideal of $R^{\prime}$.

Theorem 15 Let $(\varphi, f)$ be a onto $\left(\Gamma, \Gamma^{\prime}\right)$-homomorphism of $(R,+, \Gamma)$ and $\left(\mathrm{R}^{\prime},+, \Gamma^{\prime}\right)$ respectively and let $\mathrm{B}$ be a $\mathrm{T}-(\lambda, \mu)$-fuzzy bi-hyperideal of $\mathrm{R}^{\prime}$. Then $\varphi^{-1}(\mathrm{~B})$ is a $\mathrm{T}-(\lambda, \mu)$-fuzzy bi-hyperideal of $\mathrm{R}$.

Proof. (1) Suppose that $x, y \in R$ and $\gamma \in \Gamma$. Then we have

$$
\inf _{z \in x-y}\left\{\varphi^{-1}(B)(z)\right\} \vee \lambda=\inf _{z \in X-y}\{B((\varphi)(z))\} \vee \lambda
$$




$$
\begin{aligned}
& \inf _{\varphi(z) \in \varphi(x)-\varphi(y)}\{\mathrm{B}((\varphi)(z))\} \vee \lambda \\
& \geq \mathrm{T}(\mathrm{T}(\mathrm{B}((\varphi)(x)), \mathrm{B}((\varphi)(y))), \mu) \\
& =\mathrm{T}\left(\mathrm{T}\left(\left(\varphi^{-1}(\mathrm{~B})\right)(x),\left(\varphi^{-1}(\mathrm{~B})\right)(y)\right), \mu\right)
\end{aligned}
$$

Therefore, $\inf _{z \in x-y}\left\{\varphi^{-1}(B)(z)\right\} \vee \lambda \geq T\left(T\left(\left(\varphi^{-1}(B)\right)(x),\left(\varphi^{-1}(B)\right)(y)\right), \mu\right)$.

(2) Similarly we can prove that

$$
\inf _{a \in x \alpha y \beta z}\left(\varphi^{-1}(B)\right)(a) \vee \lambda \geq T\left(T\left(\left(\varphi^{-1}(B)\right)(x),\left(\varphi^{-1}(B)\right)(z)\right), \mu\right) .
$$

Hence $\varphi^{-1}(\mathrm{~B})$ is a $\mathrm{T}-(\lambda, \mu)$-fuzzy bi-hyperideal of $\mathrm{R}$.

\section{Conclusions}

In this paper, we have studied the $\Gamma$-hyperrings via $T$-fuzzy left and right hyperideals, T-fuzzy quasi-hyperideal and bi-hyperideal and some related properties were investigated. As a future work, one can extend these results applying the intuitionistic fuzzy theory and also to extend these results in other algebraic hyperstructure such as $(m, n)$-hyperrings etc.

\section{References}

[1] J. M. Anthony, H. Sherwood, Fuzzy groups redefined, J. Math. Anal. Appl., 69 (1979), 124-130.

[2] R. Ameri and T. Nozari, A new characterization of fundamental relation on hyperrings, Int. J. Contemp. Math. Sci., 5 (13-16) (2010), 721-738.

[3] R. Ameri and N. Shafiiyan, Fuzzy prime and primary hyperideals in hyperrings, Adv. Fuzzy Math., 2 (2007), 83-99.

[4] R. Ameri, H. Hedayati and A. Molaee, On fuzzy hyperideals in $\Gamma$ hyperrings, Iranian J. Fuzzy Syst., 6 (2) (2009), 47-59.

[5] A. Asokkumar and M. Velrajan, Characterizations of regular hyperrings, Ital. J. Pure Appl. Math., 22 (2007), 115-124.

[6] A. Asokkumar and M. Velrajan, Hyperring of matrices over a regular hyperring, Ital. J. Pure Appl. Math., 23 (2008), 113-120. 
[7] A. R. Barghi, A class of hyperrings, J. Discrete Math. Sci. Cryptogr., 6 (2-3) (2003), 227-233.

[8] W.E. Barnes, On the Г-rings of Nabusawa, Pacific J. Math., 18 (1966), 411-422.

[9] P. Corsini, Prolegomena of hypergroup theory, Supplement to Riv. Mat. Pura Appl. Aviani Editore, Tricesimo, 1993. 215 pp. ISBN: 88-7772-025-5.

[10] P. Corsini and V. Leoreanu, Applications of hyperstructure theory, Advances in Mathematics (Dordrecht), 5. Kluwer Academic Publishers, Dordrecht, 2003. xii+322 pp. ISBN: 1-4020-1222-5.

[11] P. Corsini, Hypergroupes reguliers et hypermodules, Ann. Univ. Ferrara Sez. VII (N.S.), 20 (1975), 121-135.

[12] V. Dasic, Hypernear-rings. Algebraic Hyperstructures and Applications (Xanthi, 1990), 75-85, World Sci. Publ., Teaneck, NJ, 1991.

[13] B. Davvaz, A. Salasi, A realization of hyperrings, Commun. Algebra, 34 (12) (2006), 4389-4400.

[14] B. Davvaz, Isomorphism theorems of hyperrings, Indian J. Pure Appl. Math., 35 (3) (2004), 321-331.

[15] B. Davvaz and V. Leoreanu-Fotea, Hyperring theory and applications, International Academic Press, Palm Harbor, Fla, USA, 2007.

[16] B. Davvaz, I. Cristea, Fuzzy algebraic hyperstructures. An introduction. Studies in Fuzziness and Soft Computing 321. Cham: Springer (ISBN 978-3-319-14761-1/hbk; 978-3-319-14762-8/ebook). x, 242 p.(2015).

[17] B. Davvaz, Fuzzy hyperideals in semihypergroups, Italian J. Pure Appl. Math., 8 (2000), 67-74.

[18] B. Davvaz, Fuzzy hyperideals in ternary semihyperrings, Iranian J. Fuzzy Systems, 6 (2009), 21-36.

[19] V. M. Gontineac, On hypernear-rings and H-hypergroups, Algebraic Hyperstructures and Applications, pp. 171-179, Hadronic Press, Palm Harbor, Fla, USA, 1994.

[20] K. Hila and S. Abdullah, A study on intuitionistic fuzzy sets in $\Gamma$ semihypergroups, J. Intell. Fuzzy Systems, 26 (4) (2014), 1695-1710. 
[21] E. P. Klement, R. Mesiar, and E. Pap, Triangular Norms. Trends in LogicStudia Logica Library, 8. Kluwer Academic Publishers, Dordrecht, 2000. xx+385 pp. ISBN:0-7923-6416-3.

[22] M. Krasner, A class of hyperrings and hyperfields, Int. J. Math. Math. Sci., 6 (2) (1983), 307-311.

[23] V. Leoreanu-Fotea, B. Davvaz, Fuzzy hyperrings, Fuzzy Sets and Systems, 160 (2009), 2366-2378.

[24] V. Leoreanu-Fotea, B. Davvaz, Join n-spaces and lattices, J. Mult.-Valued Logic Soft Comput., 15 (5-6) (2009), 421-432.

[25] V. Leoreanu-Fotea, B. Davvaz, n-hypergroups and binary relations, Eur. J. Combinatorics, 29 (2008), 1207-1218.

[26] C. G. Massouros, Quasicanonical hypergroups. Algebraic Hyperstructures and Applications (Xanthi, 1990), 129-136, World Sci. Publ., Teaneck, NJ, 1991.

[27] J. Mittas, Hypergroupes canoniques, Math. Balkanica, 2 (1972), 165-179.

[28] J. Mittas, Hyperanneaux et certaines de leurs proprietes, C. R. Acad. Sci. Paris Sér. A-B 2691969 A623-A626.

[29] F. Marty, Sur une generalization de la notion de group, Proceedings of the 8th Congres Math. Scandinaves, Stockholm, Sweden, (1934), 45-49.

[30] S. Mirvakili, B. Davvaz, Relations on Krasner (m, n)-hyperrings, Eur. J. Combinatorics, 31 (2010), 790-802.

[31] N. Nobusawa, On a generalization of the ring theory, Osaka J. Math., 1 (1964), 81-89.

[32] S. Ostadhadi-Dehkordi and B. Davvaz, Ideal theory in $\Gamma$-semihyperrings, Iran. J. Sci. Technol. Trans. A Sci., 37 (3) (2013), 251-263.

[33] W. Prenowitz, J. Jantosciak, Join Geometries, Springer UTM, 1979.

[34] S. Pianskool, W. Hemakul and S. Chaopraknoi, On homomorphisms of some multiplicative hyperrings, Southeast Asian Bull. Math., 32 (5) (2008), 951-958. 
[35] R. Rota, Strongly distributive multiplicative hyperrings, J. Geom., 39 (1-2) (1990), 130-138.

[36] A. Rosenfeld, Fuzzy groups, J. Math. Anal. Appl., 35 (1971), 512-517.

[37] M. De Salvo, Hyperrings and hyperfields, Ann. Sci. Univ. ClermontFerrand II Math., 22 (1984), 89-107.

[38] M. K. Sen and U. Dasgupta, Hypersemiring, Bull. Calcutta Math. Soc., 100 (2) (2008), 143-156.

[39] B. Schweizer and A. Sklar, A Statistical Metric spaces, Pacific J. Math., 10 (1) (1960), 313-334.

[40] D. Stratigopoulos, Certaines classes d'hypercorps et d'hyperanneaux Hypergroups, other multivalued structures and their applications (Udine, 1985), 105-110, Univ. Studi Udine, Udine, 1985.

[41] T. Vougiouklis, Hyperstructures and their representations, Hadronic Press Monographs in Mathematics. Hadronic Press, Inc., Palm Harbor, FL, 1994. vi+180 pp. ISBN: 0-911767-76-2.

[42] T. Vougiouklis, The fundamental relation in hyperrings. The general hyperfield. Algebraic Hyperstructures and Applications (Xanthi, 1990), 203211, World Sci. Publ., Teaneck, NJ, 1991.

[43] X. Ma, J. Zhan and V. Leoreanu-Fotea, On (fuzzy) isomorphism theorems of Г-hyperrings, Comput. Math. Appl., 60 (9) (2010), 2594-2600.

[44] M. M. Zahedi, R. Ameri, On the prime, primary and maximal subhypermodules, Ital. J. Pure Appl. Math. ,5 (1999), 61-80.

[45] L. A. Zadeh, Fuzzy sets, Inform. Control, 8 (1965), 338-353. 